\title{
"The Dementia Diva Strikes Again!": A Thematic Analysis of How Informal Carers of Persons with Dementia Use TikTok
}

\author{
Shiroq Al-Megren \\ shiroq@mit.edu \\ Mechanical Engineering Department, \\ Massachusetts Institute of Technology \\ Cambridge, USA
}

\author{
Khalid Majrashi \\ Department of Information \\ Technology, Institute of Public \\ Administration \\ Riyadh, Saudi Arabia
}

\author{
Ragad Allwihan \\ University Pre-Professional Program, \\ King Saud bin Abdulaziz University \\ for Health Sciences, King Abdullah \\ International Medical Research \\ Center \\ Jeddah, Saudi Arabia
}

\begin{abstract}
Informal carers of persons with dementia often resort to social media to alleviate their sense of social isolation and cultivate their platform to share their experience in care. The present study performed a preliminary analysis on how TikTok creators share their personal experience caring for a loved one with dementia through content shared under the hashtag \#dementiacaregiver. We performed a systemic review and inductive thematic analysis of 447 TikTok posts. The content under \#dementiacaregiver was interpreted to form five primary themes: (1) realities of caregiving, (2) a little levity, (3) advice for caring, (4) engagement with viewers, and (5) sensory stimulation. TikTok seems to have provided carers with a tool for artistic and social expression that fostered a sense of community and a place for remote belonging.
\end{abstract}

\section{CCS CONCEPTS}

- Human-centered computing $\rightarrow$ Empirical studies in collaborative and social computing.

\section{KEYWORDS}

Family caregivers; Informal caregivers; Dementia; Alzheimer's; TikTok; Thematic analysis; Inductive analysis; Social media use.

\section{ACM Reference Format:}

Shiroq Al-Megren, Khalid Majrashi, and Ragad Allwihan. 2021. "The Dementia Diva Strikes Again!": A Thematic Analysis of How Informal Carers of Persons with Dementia Use TikTok. In Mobile HCI '21: ACM International Conference on Mobile Human-Computer Interaction, Sep 03 - Oct 01, 2021. ACM, New York, NY, USA, 6 pages. https://doi.org/10.1145/3447527.3474857

\section{INTRODUCTION}

An informal carer, also known as family or unpaid carer, is a person who helps a spouse, partner, family member or friend who is in need of personal and/or functional assistance motivated by the personal relation rather than financial compensation. According to the National Alliance for Caregiving (NAC), almost one-fifth of all Americans provide informal care to an adult with health or

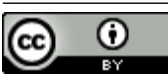

This work is licensed under a Creative Commons Attribution International 4.0 License.

Mobile HCI '21, Sep 27 - Oct 01, 2021, Toulouse, France

(c) 2021 Copyright held by the owner/author(s)

ACM ISBN 978-1-4503-8329-5/21/09.

https://doi.org/10.1145/3447527.3474857 functional needs in 2020 [7]. With ageing being one of the most important contemporary trends, it is expected to have dramatic effects on populations and health care pressures on nations, as well as on family care [5]. Dementia, characterized by a decline in memory and other cognitive functions that impairs daily activities, is a common geriatric condition that is expected to grow in prevalence as the world's elderly population grows, influencing the consequences of caring on carers.

The NAC reports that informal carers of those living with Alzheimer's or dementia assist with more instrumental everyday tasks than their counterparts, despite the increased instances of paid help [7]. The literature abounds with studies demonstrating the negative impact of care duties on carers [11], including physical, mental, social, and financial stressors, as well as the stigma associated with dementia. Caregivers often provide care for many years as the disease worsens, and these stressors may become more intense over time [20]. Caregivers also encounter changes in their lives as a result of these responsibilities, which affect their social networks and exacerbates social isolation [12].

Social media has reshaped networking behaviours by increasing individuals' connectivity and allowing users to participate directly. Informal carers have used a variety of social media tools (such as social networking sites and blogs) to share their daily care practices. The literature frequently mentions the effectiveness of social media use in supporting populations of informal caregivers of loved ones with dementia. Blogs have commonly been a source for researchers to examine the care sharing practices of caregivers of persons with Alzheimer's or dementia [2], explore caregiver's selfcare approaches [3], and analyse the needs and concerns of caregivers [16]. Another social platform, Facebook groups, have been found to provide health-related information for informal carers and encourage mental health well-being. They may be particularly advantageous for caregivers of persons diagnosed with dementia [14]. Research on dementia caregivers may benefit from a focus on online social support as a means of accessing caregivers and treatment delivery $[5,14]$. On Twitter, sentiment analysis of tweets by caregivers of loved ones with dementia was conducted to assess their experiences, which were largely negative proving the burden associated with caregiving [1].

Since its launch in 2017, TikTok, has become one of the fastestgrowing social media platforms with almost 700 million global active users as of January 2021 and is the most downloaded application in 2021's first quarter [10]. The platform offers a unique method of sharing creative short videos or audiovisual content and 
has amassed users of varied demographics (18-29:48\%; 30-49:22\%; $50-64: 14 \% ; 65+: 4 \%$ [4]; $47 \%$ of caregivers are $18-49$ years of age [7]) and covering countless topics. TikTok allows its creators to create and share short-form (15-60 second) videos while offering video editing filters and the ability to dub sound onto video. Creators can also add captions and hashtags to their videos that connects the content to a specific topic or theme. Unlike other social medial platforms, TikTok's default mode of encountering content is an algorithmically curated feed titled "For You" that is based on videos that the user have demonstrated that they want to watch. The user can refresh the content feed at any time to view new personalized selections and they can scroll through videos for hours without an "end" to the content.

The growth of TikTok, its fairly age-distributed audience, the ease of producing content via mobile phones, and the increased activity of informal carers are of interest when considering social media as a window into the caregiving experience. In this paper, we contribute to the growing literature on informal carers and their social media use by examining how TikTok creators share their personal experience caring for a loved one with dementia through content shared under the hashtag \#dementiacaregiver.

\section{METHODOLOGY}

The hashtag is a common methodological tool for data selection in online research [15]. We analysed Tiktok posts from caregivers of relatives with dementia under the hashtag \#dementiacaregiver. This hashtag was one of eight hashtags related to caregiving on TikTok that was examined by the study team to identify the hashtag most relevant to caregiving and dementia. Those hashtags were, in order of number of views, \#caregiver, \#cargiverlife, \#cargiving, \#dementiacaregiver, \#caregiveroftiktok, \#dementiacarer, \#caregiverlife, and \#dementiacarer. Our research question is focused on family or informal carers of relatives or friends with dementia, and thus the first 50 videos for each hashtag were viewed by two members of the study team to determine relevance. The hashtag \#dementacaregiver was selected for data collection as it yielded the most Tiktoks pertaining to the research question. The first 500 videos under the hashtag \#dementiacaregiver were retrieved on November 23rd, 2020.

It is worth noting that content tagged with hashtags is not displayed chronologically, but rather according to a complex algorithm that considers post and author popularity, pre-defined interests on video categories interaction, and the user's region (i.e. TikTok application). As a result, a new account was created without any categories or video interests being selected during the registration process. The research team used the new account to retrieve videos that had had no prior likes or engagements. This is to ensure that the TikTok algorithm is not influenced and hence increasing the likelihood of receiving unbiased content.

We conducted an inductive thematic analysis to identify patterns across the retrieved TikToks [6]. We follow the procedure of Herrick et al. [9] to ensure that the study is methodologically sound, given the uniqueness of TikToks as data. This began with a first step of becoming familiar with the data and constructing a preliminary codebook. In this step, two members of the study team watched the 500 retrieved TikTok posts a total of 3 times. During this time, the following exclusion criteria were applied by the authors: paid caregivers, replies to posts, duets to posts, and non-English posts. At the first viewing, 44 videos were excluded, on the second 6 more were added to the list for exclusion, and by the last viewing, an additional 3 were removed. This left us with 447 videos for analysis.

Two members of our research team separately coded the first 50 TikToks of the 447. Following that, the two researchers met to discuss the codes and to overcome any discrepancies, and update the codebook. This process was repeated until all 447 TikToks were coded and discussed. Subsequently, the researchers established similarities and inconsistencies across the codes in order to inform their categorization into themes. Five themes were potentially identified by the authors, which were then revised and the codes evaluated to ensure that they are correctly encapsulated within the themes. The length of the TikToks greatly reduced the chance of a video demonstrating mixed themes as the retrieved content ranged from 28 seconds to 60 seconds. The research team members also discussed the subthemes under the first main theme to determine if the subthemes can only be subsumed under the first main theme, and that each subtheme's substantially different from the other subthemes and cannot be grouped. To give the reader the ability to generate an understanding and a feeling as if he or she has interacted with the original videos, we expressly described the themes and chose TikToks and captions that embodied each theme prior to writing up our findings.

We believe it is necessary to highlight the following considerations about the ethical nature of data collection and use. Due to the fact that the social media posts were made public, they are considered to be in the public domain, and permission from the creators was not deemed essential for this work. The technique taken in this study is consistent with Netnography [13] and with the Ethical Guidelines for Internet Research 3.0 [8]. Additionally, in order to protect the authors' identity, we do not include any personally identifiable information in our findings, such as user names, names, or screenshots. Finally, because our data is scraped manually and not through automatic systems or software, and is not utilized for commercial purposes, we feel we adhered with TikTok's terms of service at the time of writing.

\section{FINDINGS}

We interpreted five primary themes based on the content shared with the hashtag \#dementiacaregiver. We present the themes in order of the number of posts per theme: (1) realities of caregiving (179 videos), (2) a little levity (131 videos), (3) advice for caring (71 videos), (4) engagement with viewers (52 videos), and (5) sensory stimulation (14 videos).

\subsection{Realities of Caregiving}

To illustrate the reality of caring for families with dementia, creators shared various facets of caregiving. Three subthemes mirrored this: (1) in a day, (2) the good, the bad, and the ugly, and (3) it gets worse.

3.1.1 In a Day. Several creators chose to communicate everyday activities with their loved ones, such as lunchtime, nature walks, therapy lessons, and administrating drugs. There can be significant 
value to these videos as simple everyday tasks often become daunting challenges for both seniors and their caregivers. Tips offered by creators might maximise care for others.

One creator's lunchtime videos with her mother were popular on TikTok, appearing several times under the hashtag \#dementiacaregiver. The creator is shown serving her mother breakfast, a bowl of honey bunches of oats and soy milk with blueberries, as she talks about her day. The TikTok cuts to the mother clinking her spoon against her bowl, the creator says, "Hey guys, so mom is also really tired this morning and having a hard time staying focused on eating. So I have had to stop her several times from doing some ASMR [Autonomous Sensory Meridian Response] and chewing on her spoon. So, to show you I basically just keep touching her hand and reminding her, like 'hey mom, eat your cereal' and she gets back to it." She explains that she supervises her mother's eating for that reason, as well as to keep an eye out for chocking. In another video, to keep from chocking, the creator is seen using her hand to block her mother from taking another bite while still chewing on the previous one.

Several creators posted videos of themselves reading with their loved ones. Some read children's books, while others read books that were produced for people with dementia such as "A Dusting of Snow" and the "Simple Pleasures for Special Seniors" series. One creator shows her mother reading a book with the superimposed text, "Mom loves this book written for dementia patients." Another creator communicates, "Mom used to love reading, but got discouraged because she couldn't hold onto the story. A friend suggested a children's book, so I had to get a silly one." The creator then cuts to her mother laughing while reading a children's book.

3.1.2 The Good, the Bad, and the Ugly. A few creators posted TikToks that discussed both the positive and negative sides of caring for a loved one who has dementia. These types of videos always conclude on an optimistic note, focusing on the honor of 'giving back.'

One creator cares for her father-in-law along with her husband. In one of her videos, she posted several old photos of her father-inlaw, some with her husband, as she relays, " Your parents dreamed of you before you were even born. They scarified. They fed you. They bathed you. They lived their life for you. And yeah, they probably made lots of mistakes. And one day sooner than you'd like roles will reverse." The creator then cuts to recent videos of her father-in-law and continues to share, "And they may not remember your name or how to dress on their own. But if you're up for it. It will be sad. It will be funny. It will exhausting. And it will be a blessing because God has chosen you! And you may resent some days how hard it really is. But you have the strength to make them feel loved. And keep them safe and everyone deserves the gift of dignity."

Another creator is seen in her bathroom, where she states, "sometimes the only place I can be alone". A text explains that the creator is the sole, unpaid full-time carer for her mother who has Alzheimer's The creator spoke to the camera, "If you are a caregiver then you know that appreciated is not a feeling we are used to feeling very often. And if you're not a caregiver, it is hard to grasp how lonely and isolating our jobs can be sometimes. My mom is my boss, you know. My work day starts with a dirty diaper and an hour of cleaning and consoling my boss. My boss gets violent and scared and sometimes doesn't recognise me. It's also the most satisfying job I have ever had. To be a caregiver you have to be the most selfless, patient version of yourself."

3.1.3 It Gets Worse. Select TikTok creators shared the grim reality of dementia progression. One creator shares a video of her grandmother whom she cares for wearing a backpack. The creator is heard saying, "You just wanted to put your backpack on today?" Her grandmother appears confused and replies, "I am only five years old." A text explains, "This is a difficult post. This is a video from 2 years ago when grama [sic] was able to still talk and speak somewhat clearly. During these times I also didn't understand the severity of her condition.In this video, she states that she is only five years old." The creator then follows with advice, "I hope this video can encourage someone that is caring for their loved one with dementia get educated as early as possible to know what to expect."

\subsection{A Little Levity}

For levity, some creators resorted to popular video formats on TikTok. On creator participated in a bait-and-switch trend where she shows her mother who has dementia and her father dancing to the slowed-down cover of Beach Bunny's "Cloud 9". The song starts with the premise "I hate all men", then goes into the lyrics, "But when he loves me, I feel like I'm floating." Another TikTok trend has creators sharing the biggest "adult problems" that no one prepared them for, which are often relatable. One carer uses the stitch video trend to show that caring for her mother with dementia was an "adult problem" she was not prepared for.

Another popular video format was dancing videos. One creator posted dancing TikToks of herself and her mother; in one video she was dancing while her mother was in her pajamas with text relaying, "Pretty woman pajama dance party". The same creator regularly shared 'Dementia Dance Party' videos with her mother. Another creator shows multiple clips of her mother in the same video dancing at different times of the day.

Creators also shared funny incidents with their relatives. One creator opens the door to her mother's bedrooms with a few items scattered on the bed, she says, "Welcome to my mom's art gallery full of all the things that she has collected from around the house and the should would like to put on display together." The creator spans across the room with more items scattered around and continues, "I think this is beautiful work and it is perfectly set up for the viewer and you can hear the artist in the background" The creator approaches her mother, "excuse me? are you the artists?" The mother,"No!""Oh! really? Who’s the artist then?""No!"'What was the inspiration behind your art?" The creator is seen laughing as she ends the video.

Another creator shows her mother holding a phone displaying a photo of her daughter [the creator] as a baby and as an adult. The creator asks, "Who's that baby right there?" "Uh, you." "and who's that young lady right there?" "where?" [the creator point at her adult photo and says, "Can you believe that was me as a baby?" "huh-uh" "Was I cute?" "Um...Not really." Both start laughing. 


\subsection{Advice for Caring}

Creators shared advice for caring for loved ones with dementia. One creator cares for her father who has Alzheimer's and shares a TikTok with a tip from her personal experience, "Another tip I would give is do not waste time correcting someone who has dementia." She gives an example, "like let's say my dad said 'I'm really stressed out because I need to go to a meeting.' and you know he doesn't have a meeting. You would see people correct him. They would be like 'what are you talking about. You don't have a meeting.' Don't do it, it doesn't help. It doesn't validate what they are going through. It makes them more agitated. "The creator then follows with a better approach to handle this situation, "A much much better way to handle it is to redirect them. Saying, 'oh well, that sounds frustrating that you don't have the time. Do you want to play some checkers while we wait?' Because their memory is impaired, odds are they will forget they were thinking about that. So don't delve into it and don't correct."

Another creator caring for her non-verbal grandmother with dementia shared dietary advice. In her short video, the creator emphasized the importance of having a routine especially for those who are non-verbal stating that "as time goes on they can decline and do less and less". The creator continues sharing a snack idea as she shares that her grandmother dislikes being fed but loves snacking on grapes. The creator tells her viewers that grapes "have plant compounds that may protect against certain cancers, packed with nutrients especially Vitamin $\mathrm{C}$ and $\mathrm{K}$, high antioxidant content that may prevent chronic diseases and may decrease blood sugar." The creator then follows this advice on how she presents the snack to her grandmother, e.g. frozen and at times sprinkled with a little sugar.

A number of creators spoke up in support of music's beneficial impact on their dementia-affected loved ones. One creator showed her mother engaging in dance therapy via an online class. She tells her the viewers, "Thank you to those that recommended this. I haven't seen her this active in years!" Another creator shares a TikTok of the changes in her father since she started playing music for him. The creator says there was a noticeable change in his mood, sleep, and had more "better days than bad". One creator recommends dancing with ribbon wands while listening to music. She shares that this activity is a good way to practice both large and small movements simultaneously.

\subsection{Engagement with Viewers}

Frequently, TikTok creators will end their videos by inviting viewers to ask questions about their experience as caregivers, their loved ones, or dementia. Creators would also take the time to answer questions from the Q\&A section.

A creator caring for her mother is asked, "Did your mom understand her diagnosis at first? How did she feel about it?" The creator acknowledges that she gets this question a lot and she is ready to answer it. The creator says, "My mom definitely had a hard time with her diagnosis. It took us quite a few years. Throughout that time there were different levels of understanding from my mom and there's a huge range of emotions. There was a lot of confusion, a lot of denials, anger, sadness, and all that is completely understandable because going through something like that is extremely difficult. It was a really hard time for both my mom and for my family. Denial,anger, and anxiety are very common emotions for someone going through a diagnosis of dementia or Alzheimer's and I think it's very important for the caregivers and loved ones around them to remember this to be patient and to give as much love as possible as they are going through this process."

In other TikToks, viewers were curious to learn more about resources or approaches that the writers had discussed and/or considered useful for their dementia-affected loved ones. When a creator showed off her mother's new fidget gadget she was asked, "Can you show what's on [sic] her fidget toy thing. $\mathrm{Plz}$ [sic]" The creator proceeded to show the fidget's different compartments with various areas for buttons, zippers, laces, snaps, clips, pictures, and sensory areas. The creator also continues to share another fidget that her mother has that offered different forms of sensory stimulation. The creator ends the videos by saying that fidgets are "really cool and I highly recommend them".

Additionally, some creators responded to negative comments. One viewer felt the mother with dementia was being used by the daughter and left the following comment, "I don't like these post [sic]. Why are we exploiting This [sic] people with dementia. I pray my children don't do this to [sic]" The creator states that she has her father's permission for doing these video. She also states that since her parents have been married for 50 years, the creators feel her father's permission if all she needs. The creator also insists that she knows her mother would likely be proud of her if she were well. The creator ends the video by saying, "I am using my TikTok to help myself and to help other people who have mothers or fathers with dementia. That's it. If that is the way you feel, you are entitled to your opinion."

\subsection{Sensory Stimulation}

Creators shared engaging and interactive activities for their relatives with dementia to offer fun, creative, and constructive ways to spend time with their loved ones. One creator shared a TikTok where her mother was arranging pegs according to their colours. The creator says, " She cannot do as much as other people with dementia, FTD [Frontotemporal dementia], things like that. But I wanted to pull this out and still let her fiddle with it." Another creator engaged her mother in small art projects, such as applying stickers onto picture, colouring, and scrapping. The creator shared a tip for no mess paining in one of her videos, she writes "Ziploc bag, canvas, paint, glitter" with her mother singing while engaging with the activity. The creator is heard behind the camera encouraging her mother to keep "squishing". In one video, another creator was trying to coax her mother into an art project with little interest from the mother. She says, "It's not always like this. It's different every day. I meet her where she is." with an old picture of her mother in paint speckled dungarees. Often stimulating activities were tailored to the relatives' interests and abilities.

While the majority of videos showed and offered advice on how to care for female relatives with dementia.One creator gave advice for dementia activities "just for men". The creator says, "Men won't partake in feminine activities with feminine object. Instead of Elmers glue get wood glue. Instead of tissue paper use brown butcher's paper. So adapt your material for men. Here are some 
idea for activities you can do. Polish dress shoes with shoe polish or clear polish and a brush. Or take out shoe laces from sneakers and clean them and have him re-lace everything. Get a small piece of wooden furniture and he can sand them down and re-polish them or paint them and stain them."

Fidget toys and sensory therapy have shown to be helpful in reducing agitation and anxiety in dementia patients [19]. One creator handed her mother artificial flowers for sensory play, but after a while of none engagement the creator cuts the video to her handing her mother a fidget book instead saying, "She really didn't end up doing anything with the flowers, so I gave her her fidget book." Another creator shared a video of her unboxing a number of fidget gadgets and dolls she has bought for her mother. She goes over the different fidgets and shows the different ways her mother can engage with the toy.

\section{DISCUSSION AND CONCLUSIONS}

We explored the content posted by Tiktok creators that self-identified as informal carers of loved ones with dementia under the hashtag \#dementiacaregiver. The carers utilised this platform to (1) share their realities of caregiving, (2) provide a little levity, (3) give advice for caring, (4) engage with viewers, and (5) demonstrate sensory stimulation activities and gadgets. In this section, we briefly discuss our findings.

A dominant theme in this study is the realistic portrayal of the caregiving role showcasing their day-to-day activities along with tips and the stark realities of caring for a loved one with dementia. Interestingly, while many TikTok creators use a selfie-like approach to video creation [17], this was not the case for many of the videos in this topic. Instead, creators would share the scene with a loved one and engage with them on video. This could be for a variety of reasons, including teaching purposes, such as demonstrating how to keep a person with dementia from eating too quickly. Often, these videos offer a sense of solidarity and solidify the idea that the viewer, if a caregiver, is 'not alone. For the creators, these TikToks draw attention to the unfiltered joys and burden of caring for a loved one with dementia.

TikTok thrives on humor [17]. Since, short-form video platforms are commonly used by creators for entertainment, with videos featuring jokes, hilarious moments, singing, and dancing. This has also been the case for creators under the hashtag \#dementiacaregiver. Compared with other platforms, we found that TikTok allowed carers to share moments of respite with their loved ones. The demands of caregiving are stressful and overwhelming. Cultivating strategies for emotional and physical well-being is important to prevent burnout. Humor facilitated with TikTok is a useful communication tool and permits the caregiver to cope with aversive experiences of life [18].

Creators frequently interacted with viewers, offering general advice based on what worked for the person for whom they are caring. They often encouraged participation, asking viewers to comment on videos, duet, or use the Q\&A functionality on TikTok. Questions at times were personal, most would ask for advice about caring for someone with dementia or Alzheimer's. The advice varied from therapies, tools or gadgets, foods, medicine administrations, and online guides. This constructive use of social media should be investigated further, as well as promoted as a way of offering vital online assistance, connecting those with common interests, and identifying people who could benefit from resource recommendations.

Social media platforms enabled the publicization of formerly private experiences, such as caregiver experiences. This work contributes significant insight into the unique informal caregiver experience on Tiktok by supplementing earlier research on caregivers' everyday experiences, care and support techniques (for their loved ones and for themselves), and concerns. By building a supportive online community for dementia carers, Tiktok can be used as a resource for anyone embarking on the journey of caring for a loved one who has recently been diagnosed with dementia. Additionally, it can serve as a platform for experts and health care providers to engage with such caregivers and promote self-care habits and interventions.

\section{REFERENCES}

[1] Reda Al-Bahrani, Margaret K Danilovich, Wei-Keng Liao, Alok Choudhary, and Ankit Agrawal. 2017. Analyzing Informal Caregiving Expression in Social Media. In 2017 IEEE International Conference on Data Mining Workshops (ICDMW). IEEE, 342-349.

[2] Joel G Anderson, Elizabeth Hundt, Morgan Dean, Jessica Keim-Malpass, and Ruth Palan Lopez. 2017. "The Church of Online Support" Examining the Use of Blogs Among Family Caregivers of Persons With Dementia. Journal of Family Nursing 23, 1 (2017), 34-54.

[3] Joel G Anderson, Elizabeth Hundt, Morgan Dean, and Karen M Rose. 2019. "A fine line that we walk every day": Self-care approaches used by family caregivers of persons with dementia. Issues in mental health nursing 40, 3 (2019), 252-259.

[4] Brooke Auxier and Monica Anderson. 2021. Social Media Use in 2021. https:// www.pewresearch.org/internet/2021/04/07/social-media-use-in-2021/. Accessed: 2021-03-14.

[5] Pavel Bachmann. 2020. Caregivers' Experience of Caring for a Family Member with Alzheimer's Disease: A Content Analysis of Longitudinal Social Media Communication. International fournal of Environmental Research and Public Health 17, 12 (2020), 4412.

[6] Virginia Braun and Victoria Clarke. 2006. Using thematic analysis in psychology. Qualitative research in psychology 3, 2 (2006), 77-101.

[7] National Alliance for Caregiving. 2020. Caregiving in the US 2020. https://www. caregiving.org/caregiving-in-the-us-2020/. Accessed: 2021-03-30.

[8] Aline Shakti Franzke, Anja Bechmann, Michael Zimmer, C Ess, et al. 2020. Internet research: Ethical guidelines 3.0. Association of Internet Researchers (2020).

[9] Shannon SC Herrick, Laura Hallward, and Lindsay R Duncan. 2021. "This is just how I cope": An inductive thematic analysis of eating disorder recovery content created and shared on TikTok using\# EDrecovery. International fournal of Eating Disorders 54, 4 (2021), 516-526.

[10] Hootsuite. 2021. The Global State of Digital 2021. https://www.hootsuite.com/ pages/digital-trends-2021. Accessed: 2021-03-14.

[11] Mike C Horton, Jan Oyebode, Linda Clare, Molly Megson, Leanne Shearsmith, Carol Brayne, Paul Kind, Zoe Hoare, Hareth Al Janabi, Val Hewison, et al. 2021. Measuring quality of life in carers of people with dementia: development and psychometric evaluation of scales measuring the impact of DEmentia on CARers (SIDECAR). The Gerontologist 61, 3 (2021), e1-e11.

[12] Cristina Huertas-Domingo, María Márquez-González, Isabel Cabrera, Samara Barrera-Caballero, María del Sequeros Pedroso-Chaparro, Rosa Romero-Moreno, and Andrés Losada-Baltar. 2021. Sociocultural Influences on the Feeling of Loneliness of Family Caregivers of People with Dementia: The Role of Kinship. International Fournal of Environmental Research and Public Health 18, 9 (2021), 4700.

[13] Robert V Kozinets. 2019. Netnography: The essential guide to qualitative social media research. Sage.

[14] Jenny A Lagervall, Madeline R Lag, Sophie Brickman, Rebecca E Ingram, and Leilani Feliciano. 2019. Give a piece of your mind: A content analysis of a Facebook support group for dementia caregivers. Innovation in Aging 3, Suppl 1 (2019), S850.

[15] Ioana Literat. 2021. "Teachers Act Like We're Robots": TikTok as a Window Into Youth Experiences of Online Learning During COVID-19. AERA Open 7 (2021), 2332858421995537.

[16] Susan M McLennon, Altheria Davis, Sarah Covington, and Joel G Anderson. 2019. "At the End We Feel Forgotten": Needs, Concerns, and Advice from Blogs of Dementia Family Caregivers. Clinical nursing research (2019), 1054773819865871. 
[17] Aliaksandra Shutsko. 2020. User-Generated Short Video Content in Social Media. A Case Study of TikTok. In International Conference on Human-Computer Interaction. Springer, 108-125.

[18] Lisa Sparks Bethea, Shirley S Travis, and Loretta Pecchioni. 2000. Family caregivers' use of humor in conveying information about caring for dependent older adults. Health communication 12, 4 (2000), 361-376.
[19] Benedicte S Strøm, Siri Ytrehus, and Ellen-Karine Grov. 2016. Sensory stimulation for persons with dementia: a review of the literature. Fournal of clinical nursing 25, 13-14 (2016), 1805-1834.

[20] MAG Van Den Wijngaart, MJFJ Vernooij-Dassen, and AJA Felling. 2007. The influence of stressors, appraisal and personal conditions on the burden of spousal caregivers of persons with dementia. Aging \& Mental Health 11, 6 (2007), 626-636. 